\title{
Electronic Reporting Systems in Pharmacovigilance: The Implementation of VigiFlow in Brazil
}

\author{
Marcelo Vogler ${ }^{1} \cdot$ Heloísa Ricci Conesa ${ }^{2} \cdot$ Karla de Araújo Ferreira ${ }^{1} \cdot$ Flávia Moreira Cruz $^{1}$. \\ Fernanda Simioni Gasparotto ${ }^{1} \cdot$ Karen Fleck $^{1} \cdot$ Fernanda Maciel Rebelo $^{1} \cdot$ Bianca Kollross ${ }^{1}$. \\ Yannie Silveira Gonçalves ${ }^{1}$
}

Published online: 15 September 2020

(c) The Author(s) 2020

\begin{abstract}
Pharmacovigilance is defined by the World Health Organization as the science and practice related to the detection, assessment, understanding and prevention of adverse drug reactions (ADRs). In a well-functioning pharmacovigilance system, information on possible ADRs is collected from patients, health professionals and marketing authorisation holders. Of all data sources for drug safety monitoring, spontaneous reporting systems provide the highest volume of information at the lowest maintenance cost and have proven their value in the early detection of product-related safety issues. At the beginning of 2018, the Brazilian Health Regulatory Agency (Agência Nacional de Vigilância Sanitária-Anvisa) decided to replace the national electronic reporting system in Brazil (Notivisa) with the system provided by Uppsala Monitoring Centre (VigiFlow) for ADR reporting. This paper describes that process and reports on the progress made by Anvisa in terms of making Brazil compliant with international pharmacovigilance standards as well as significantly increasing the number of individual case safety reports collected.
\end{abstract}

\section{Key Points}

Brazilian Health Regulatory Agency (Agência Nacional de Vigilância Sanitária - Anvisa) implemented VigiFlow in 2018 as an initiative to improve the national pharmacovigilance system.

Individual case study reports (ICSRs) rates increased after the implementation of Vigiflow in Brazil.

From 2020, all pharmacovigilance stakeholders will be able to report ICSRs to VigiFlow, which will expand and reinforce the national use of VigiFlow in the years to come.
Marcelo Vogler

marcelo.moraes@ anvisa.gov.br

1 Brazilian Health Regulatory Agency (Agência Nacional de Vigilância Sanitária-Anvisa), Brasília, DF, Brazil

2 Uppsala Monitoring Centre (UMC), Uppsala, Sweden

\section{Introduction}

Before medicines are approved for sale they must be tested for quality, safety and efficacy. Clinical trials in particular provide regulatory agencies with the evidence they need on safety and efficacy for market approval. However, such studies have limitations, and the information collected during the pre-marketing phase is incomplete when it comes to possible adverse drug reactions (ADRs). This is because the patients in clinical trials are selected and few in number, and studies are limited in duration. The conditions of use also differ from clinical practice and information about rare adverse reactions, chronic toxicity, use in special groups (children, elderly, pregnant women) or drug interactions are usually incomplete or unavailable [1].

Consequently, it has been necessary to establish drug monitoring mechanisms related to the detection, assessment, understanding and prevention of adverse events or any other problems related to medicines [2]. Pharmacovigilance, or medicine safety, implies a constant state of watchfulness to recognise when things are going wrong, to take action to solve immediate problems, and to share information about the causes of harm to influence future policy and practice [3]. Among the different methods of reporting suspected 
ADRs, spontaneous reporting systems provide the highest volume of information at the lowest maintenance cost and have proven their value in the early detection of drug-related problems [4].

The need for rapid information sharing, which became evident in the late 1950s and early 1960s with the thalidomide tragedy, led the World Health Organization (WHO) to organise a systematic data collection for ADRs [5]. At the end of the 1960s, a pilot project was launched with the 10 founder member countries of the WHO Programme for International Drug Monitoring (WHO PIDM) [6].

Today there are 136 countries in the WHO PIDM that share their data with VigiBase, the WHO global database of individual case study reports (ICSRs), which is maintained by Uppsala Monitoring Centre (UMC) in its role as the WHO Collaborating Centre for International Drug Monitoring. As of January 2020, VigiBase contained more than 20 million ICSRs that are used for detecting possible safety signals $[7,8]$.

In Brazil, the Ministry of Health directive No. 696 of 7 May 2001 appointed the Pharmacovigilance Office of the Brazilian Health Regulatory Agency (Agência Nacional de Vigilância Sanitária-Anvisa) as its national pharmacovigilance centre [9], and in 2007 a national spontaneous electronic reporting system - the Health Surveillance Reporting System Notivisa-was set up with its own database. As a member of the WHO PIDM since 2001, Brazil is required to send reports of suspected ADRs to VigiBase in a format compatible with the international standard for electronic transmission of ICSRs, the International Conference on Harmonisation of Technical Requirements for Registration of Pharmaceuticals for Human Use (ICH) E2B [10]. This format is a standardised structure for sending information electronically between databases and contains all the relevant data elements to assess ICSRs [11]. However, as Notivisa is not compatible with the ICH E2B format, it meant manually entering data from Notivisa to a limited, free version of VigiFlow.

In addition to not being compliant with ICH E2B, Notivisa was also limited by system instability, an inability to import data series or export the database for analysis, lack of analytical and statistical tools, and only working with the internet browser Internet Explorer.

The need for a replacement was reinforced in 2016 when Brazil joined the $\mathrm{ICH}$, which made the incorporation of $\mathrm{ICH}$ guidelines mandatory [12].

At the beginning of 2018, Anvisa decided to adopt VigiFlow as the official reporting system for ICSRs in Brazil. The aim of this paper is to present a qualitative description of the decision-making process, led by Anvisa's Pharmacovigilance Office (PVO), and the outcomes after 1 year of implementation of VigiFlow in Brazil.

\section{Decision-making}

Since the implementation of Notivisa in 2007 there had been frequent problems in its operation that required several corrections and improvements. The system was partially inoperative at the end of 2017 and beginning of 2018. Reporters were unable to gain access and the number of ICSRs fell significantly between December 2017 and February 2018.

The process of acquiring a new electronic system had already begun by that time. But the estimated tender cost was millions of US dollars and the time frame for the system to be installed and fully functional-taking into account purchase, customisation, testing, implementation, adjustments and completion-was expected to be a couple of years. It was then that PVO proposed looking into VigiFlow as an alternative.

VigiFlow is an ICSRs management system for countries that require an electronic pharmacovigilance database for the collection, processing and sharing of ICSRs for effective data analysis. It was initially developed for Switzerland's regulatory agency Swissmedic. Today it is offered to national centres in low- and middle-income countries as a low-cost way of managing their pharmacovigilance data. Previously, a free version of VigiFlow with limited functionality was also available to support countries reporting ICSRs to VigiBase.

VigiFlow is compatible with the international ICH E2B standard-and uses the international Medical Dictionary for Regulatory Activities (MedDRA) terminology_for efficient data exchange between pharmaceutical companies and regulatory agencies using $\mathrm{xml}$ files. It supports the setup of a decentralised system for data collection, allows integration with an electronic form for the general public and healthcare professionals to report adverse events with medicines or vaccines (eReporting), and offers direct sending of ICSRs to VigiBase.

Currently, VigiFlow is used by more than 90 countries worldwide. The system is continuously updated, and the latest version of VigiFlow launched in January 2018 is compliant with the updated standard ICH E2B(R3), which includes improvements in manual data entry and data structure of ADR and AEFI (adverse events following immunisation) reports, as well as better workflow support for pharmacovigilance centres. The new version of VigiFlow is the same for all countries, with a free, limited version no longer available.

To find out how VigiFlow works in detail and present its needs and requirements, Anvisa held several phone conferences with UMC, and in November 2018 five team members travelled to Uppsala, Sweden on a technical visit. The characteristics and functionalities of VigiFlow were compared with those of Notivisa and the requirements of the National Pharmacovigilance System of Brazil. Table 1 shows the 
results of the comparative analysis that justified the replacement of Notivisa by VigiFlow.

The gathering of the technical and operational requirements on which the board of directors would base their decision followed established processes and procedures for project management. It was roughly 8 months before the process of purchasing and implementing the new system could begin.

\section{Implementation}

In October 2018, Anvisa and UMC signed the licence agreement for VigiFlow and work on setting up the system began.

\subsection{VigiFlow Setup}

When implementation started, VigiFlow was available only in English, French and Spanish. The first task after the goahead had been given was to translate the system to Brazilian Portuguese. To begin with, some 395 terms and 116 help texts were translated from 11 screens, in addition to the terms of use (363 words). The PVO team also checked that they were compatible with the terms used in Notivisa to minimise the impact to users of the system change. The translation was then sent to UMC for implementation in VigiFlow.

Besides the system translation, problems relating to the proper coding of adverse events with MedDRA were also identified. The terminology already had an official Portuguese version that was implemented in VigiFlow. However, Anvisa assessed a sample of 2700 terms suggested by MedDRA Maintenance and Support Services Organization (MSSO) and almost $30 \%$ contained differences between Iberic and Brazilian Portuguese (it is worth noting here that an audit would have been necessary regardless of the technological solution adopted by Anvisa since the implementation of MedDRA is part of the action plan to implement ICH guidelines in Brazil by 2021). Following discussions with MSSO, it was decided in November 2018 that MedDRA - with support from Anvisa, which would be responsible for validating the translated terms - would produce a Brazilian Portuguese version of the terminology by March 2020.

In parallel, a decision was also taken to change the name VigiFlow to VigiMed to make it easier for Brazilians to understand. It was thought that the unfamiliarity of the term VigiFlow could hinder user acceptance, especially among the general public whose English may be limited. No technical adaptations were made to the system as a result of the name change since VigiFlow is a one-size-fits-all solution and not adjustable on a country-by-country basis.

\subsection{VigiFlow Operation}

During the implementation phase, VigiFlow was introduced alongside Notivisa to make the transition as smooth as possible. This includes the transfer of legacy data from Notivisa to VigiFlow to create a single national ICSR database (a process that was still ongoing when this paper was written). The administration process followed three steps corresponding to the number of VigiFlow components or modules to be implemented.

\subsection{1 eReporting Module}

Launched on 10 December 2018 at the same time as PVO started using VigiFlow, eReporting is accessed through an open link on Anvisa's website. This module is targeted at voluntary reporting by the general public and health professionals who are not linked to any healthcare service.

\subsubsection{Regional Centre Module}

This module was made available by UMC in February 2019. The migration of all Notivisa users to VigiFlow, including training, started in April 2019 with a pilot of six sentinel hospitals.

The major obstacle with this step was to find a solution for decentralised access to the system. As the administrative structure for healthcare is decentralised in Brazil, there was a need to create hierarchical access profiles based on the public Unified Health System (UHS). Accordingly, three hierarchical levels were created within VigiFlow, as follows:

- Anvisa: highest hierarchical level, corresponding to the complexity of pharmacovigilance activities, with access to all system functionality and all ICSRs created by other levels. As Brazil's national pharmacovigilance centre, Anvisa is responsible for sending ICSRs to VigiBase once they have been assessed.

- State Health Surveillance units (SHSUs): hierarchical level immediately below Anvisa, considered as regional centres with access to their own ICSRs and local healthcare services, including hospitals from the Sentinel Network that actively monitor the products regulated by Anvisa [13].

- Healthcare services, including hospitals from the Sentinel Network: lowest hierarchical level, comprising healthcare units legally obligated to have a Patient Safety Unit in their organisational structure. These are also classed as regional centres but are subordinate to State Health Surveillance units according to their geographical location. Each healthcare service has access only to its own ICSRs. 


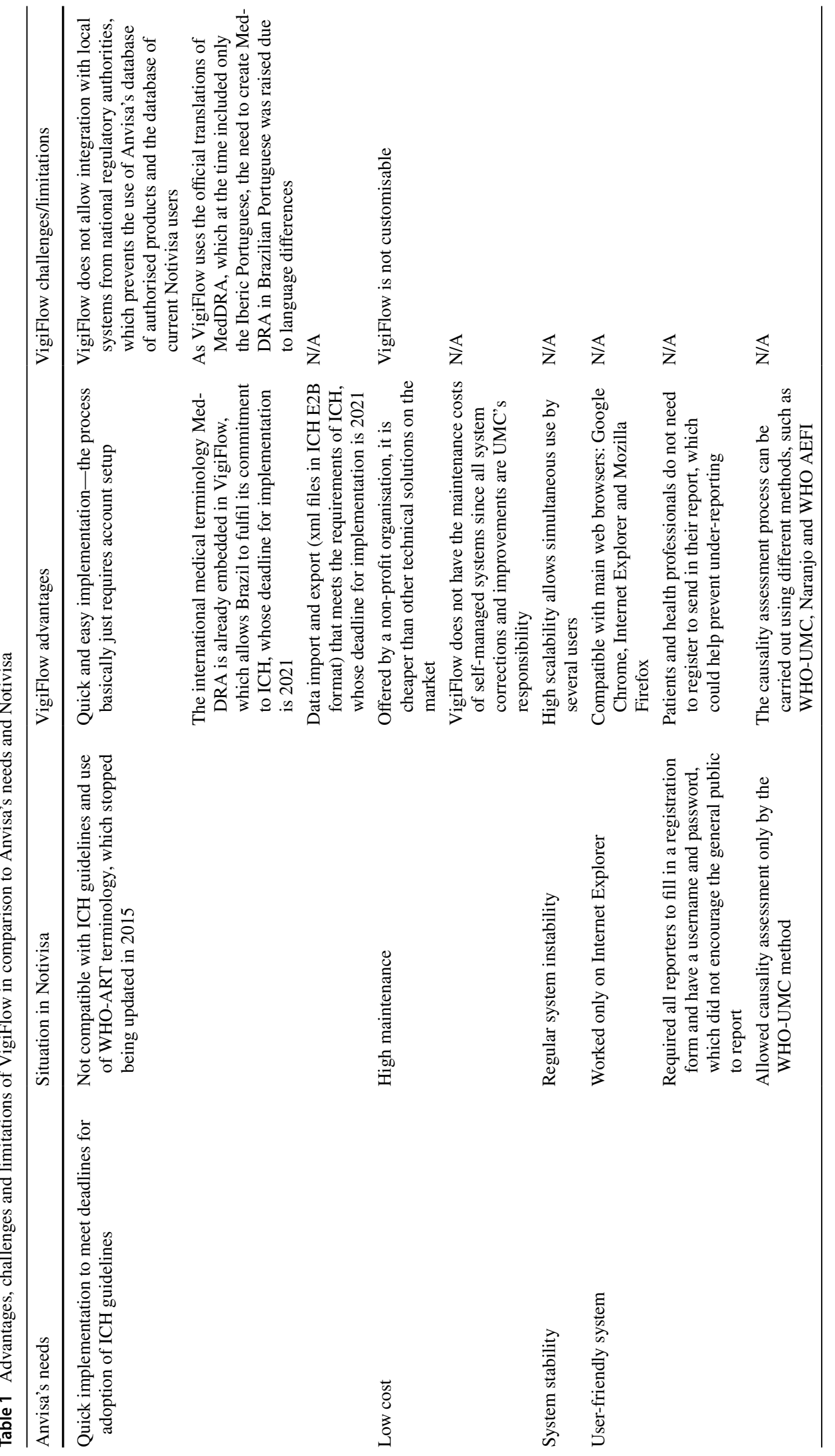




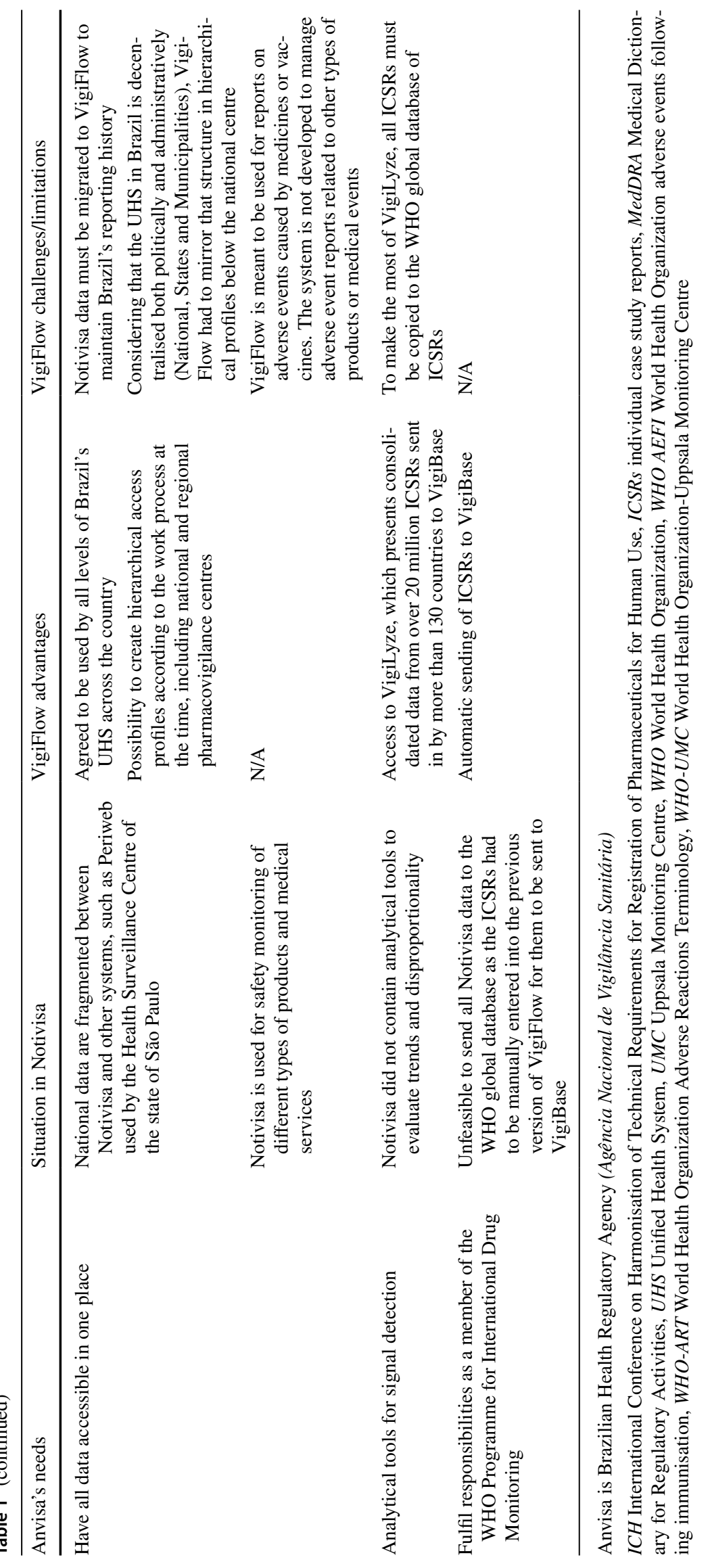




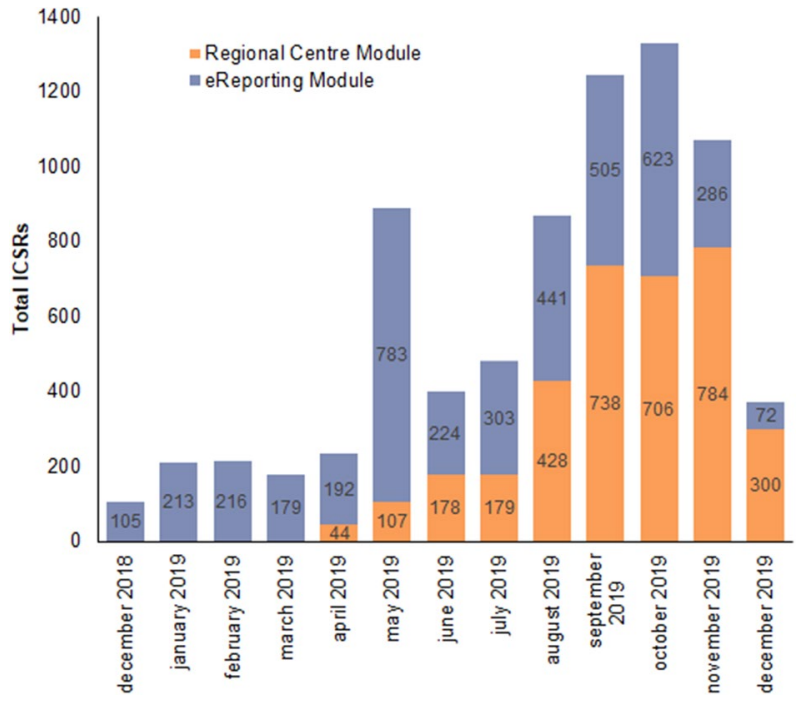

Fig. 1 Total individual case study reports (ICSRs) received per month by Anvisa in VigiFlow between 10 December 2018 and 10 December 2019 grouped by reporting method (regional centre or eReporting module). Anvisa is Brazilian Health Regulatory Agency (Agência Nacional de Vigilância Sanitária)

To make the transition process easier for regional centres, Anvisa established a communication and training plan. First, PVO created an e-mail address for requesting user accounts and general questions. Additionally, an FAQ was compiled during the pilot phase with the six sentinel hospitals. Later, training was provided via webinars and printed materials.

\subsubsection{Marketing Authorisation Holder Module}

This module is planned for 2020. It will be pharmaceutical companies' responsibility to upload to the system xml files in ICH E2B. Companies whose systems are not compatible with the standard will have to submit their ICSRs manually in VigiFlow. Marketing authorisation holders must follow the specific regulatory requirements for reporting [14].

\section{Outcomes}

\subsection{Number of ICSRs}

Data gathered between 10 December 2018 (the date the eReporting VigiFlow module started to be used) and 10 December 2019 showed that 7606 ICSRs were received, with 4142 (54.5\%) coming from the eReporting module (Fig. 1).

Making VigiFlow available for ADR reporting alongside Notivisa led to a $62.6 \%$ increase in ICSRs received by Anvisa compared to the same period for the previous year (2018 vs 2019). Figure 2 shows the volume of ICSRs received by both systems in 2019. Access to Notivisa for ADR reporting will be blocked for everyone except marketing authorisation holders from January 2020.

It should be noted that due to the use of VigiFlow, 100 $\%$ of ADR reports were sent to VigiBase in 2019 with a causality assessment compared with only $10 \%$ of the ICSRs collected in Notivisa.
Fig. 2 Total individual case study reports (ICSRs) received per month and per year by Anvisa through VigiFlow and Notivisa. Anvisa is Brazilian Health Regulatory Agency (Agência Nacional de Vigilância Sanitária)

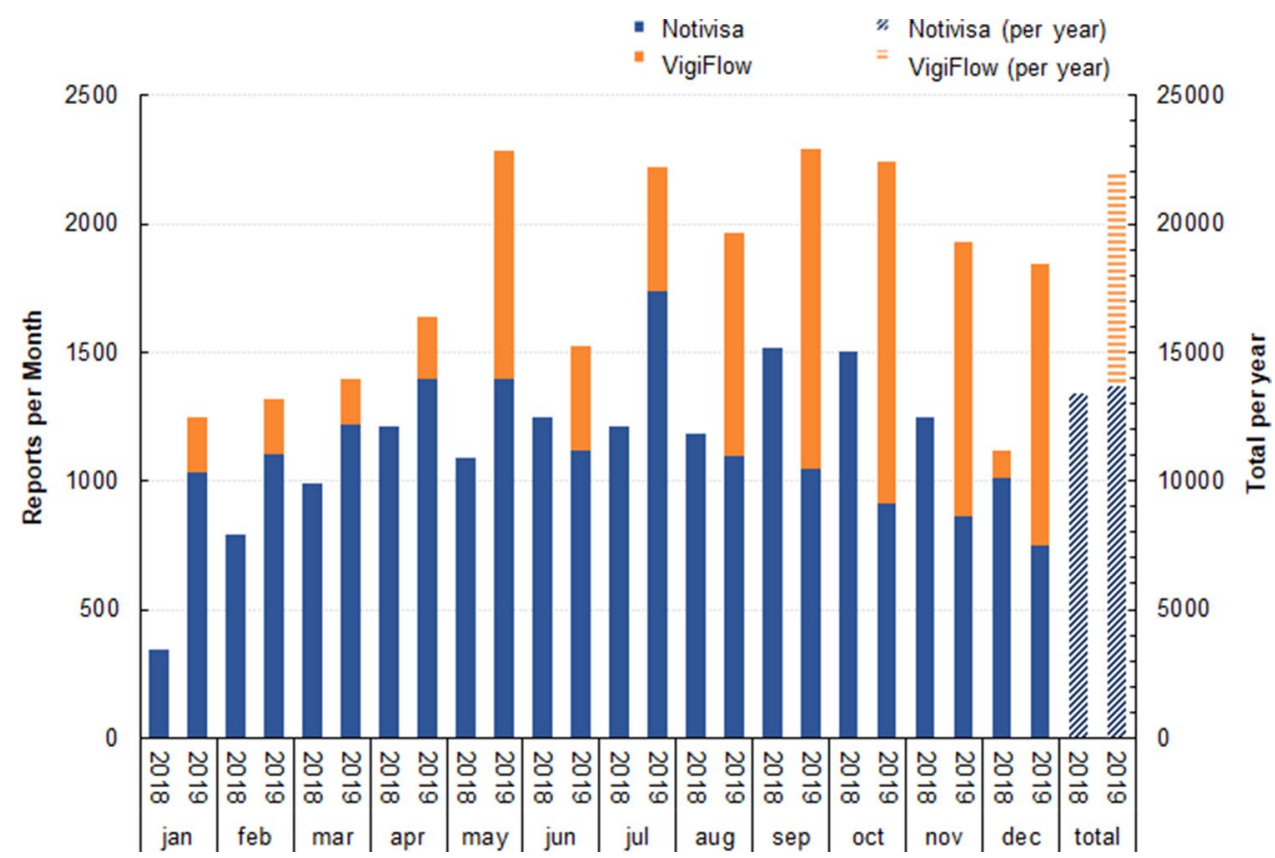




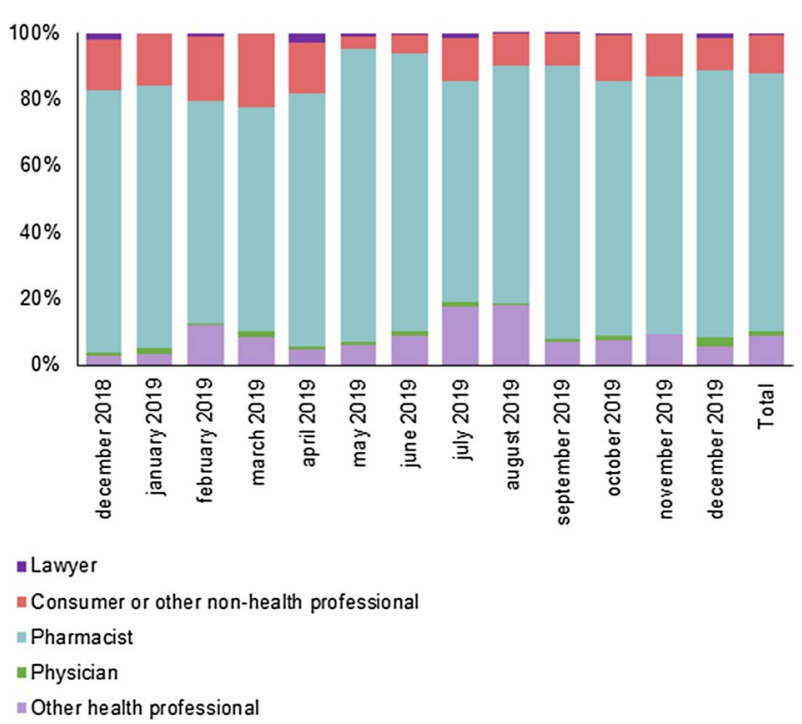

Fig. 3 Percentage of individual case study reports (ICSRs) received per month by Anvisa in VigiFlow between 10 December 2018 and 10 December 2019 by reporter group. Anvisa is Brazilian Health Regulatory Agency (Agência Nacional de Vigilância Sanitária)

Pharmacists are the biggest reporter group, sending $78 \%$ of all ICSRs (Fig. 3).

Since VigiFlow's introduction, there has been a significant increase in the number of reports from the general public. In the period analysed, reports more than doubled from consumers or non-health professionals compared with the same period a year earlier (up from 200 reports in Notivisa to 467). This suggests that the eReporting tool is easier to use for people not familiar with pharmacovigilance concepts.

\subsection{Promotion and Training}

The increased number of ICSRs received in 2019 can also be attributed to promotion and training. In May 2019, the first webinar on eReporting targeted at members of the general public and independent health professionals had 354 participants. In June 2019, a second webinar targeted at healthcare services had 295 participants.

Additionally, Anvisa has promoted VigiFlow at scientific congresses and technical events organised by stakeholders or patient associations for health professionals or patients. And it has used articles and social media to talk about pharmacovigilance and VigiFlow to the general public and health professionals.

Promotional efforts have been directed at SHSUs and healthcare services aiming to discontinue access to Notivisa, too. As of December 2019, 1.945 users from 667 SHSUs / healthcare services were registered in VigiFlow.

\section{Final Considerations}

Brazil is a country of continental dimensions, with over 200 million inhabitants living in more than 5000 municipalities across 27 states. This makes the implementation of any national electronic system a challenge. While there were benefits to using VigiFlow for Anvisa, adopting a fixed system with no possibility of customisation also meant obstacles had to be overcome.

However, with the system functioning without interruption since it went live, its implementation can be said to be a success. Based on the improvements suggested by Brazil, VigiFlow has demonstrated that it meets the needs of the Brazilian Pharmacovigilance System.

Notivisa is due to completely shut down in the second half of 2020, making VigiFlow the only platform by which all stakeholders, including marketing authorisation holders, can report adverse drug events. It is expected that, through awareness promotion, the use of VigiFlow in Brazil will expand with more healthcare services and users registered, increasing the number of reports by inhabitants and reinforcing national pharmacovigilance in the years to come.

Acknowledgements The authors would like to thank Graeme Nadasy from the Global Communications department at the Uppsala Monitoring Centre for his support in reviewing this article.

\section{Declarations}

Funding No funding was received.

Conflict of Interest The authors have no conflicts of interest to report.

Ethical Approval Not applicable.

Consent to Participate Not applicable.

Consent for Publication Not applicable.

Availability of Data and Material Not applicable.

Code Availability Not applicable.

Author Contributions All authors contributed to the study conception and design. Data collection and analysis were performed by Karla de Araújo Ferreira and Yannie Silveira Gonçalves. The first draft of the manuscript was written by Marcelo Vogler and Heloísa Ricci Conesa, and all authors commented on previous versions of the manuscript. All authors read and approved the final manuscript.

Open Access This article is licensed under a Creative Commons Attribution-NonCommercial 4.0 International License, which permits any non-commercial use, sharing, adaptation, distribution and reproduction in any medium or format, as long as you give appropriate credit to the original author(s) and the source, provide a link to the Creative Commons licence, and indicate if changes were made. The images or other third party material in this article are included in the article's Creative Commons licence, unless indicated otherwise in a credit line to the 
material. If material is not included in the article's Creative Commons licence and your intended use is not permitted by statutory regulation or exceeds the permitted use, you will need to obtain permission directly from the copyright holder. To view a copy of this licence, visit http://creativecommons.org/licenses/by-nc/4.0/.

\section{References}

1. Uppsala Monitoring Centre, World Health Organization. Safety monitoring of medicinal products: guidelines for setting up and running a pharmacovigilance centre. Uppsala: Uppsala Monitoring Centre; 2000.

2. World Health Organization. Pharmacovigilance. 2020. https:// www.who.int/medicines/areas/quality_safety/safety_efficacy/ pharmvigi/en/. Accessed 4 Mar 2020.

3. Uppsala Monitoring Centre. 50 years of pharmacovigilance. 2020. https://www.who-umc.org/global-pharmacovigilance/globa 1-pharmacovigilance/50-years-of-pharmacovigilance/. Accessed 4 Mar 2020.

4. Pal SN, Duncombe C, Falzon D, Olsson S. WHO strategy for collecting safety data in public health programmes: complementing spontaneous reporting systems. Drug Saf. 2013;36:75-81.

5. Uppsala Monitoring Centre. Making medicines safer: Uppsala Monitoring Centre-40 years of pioneering pharmacovigilance. Uppsala: Uppsala Monitoring Centre; 2018.

6. Venulet J, Helling-Borda M. WHO's International Drug Monitoring - the formative years, 1968-1975: preparatory, pilot and early operational phases. Drug Saf. 2010;33:e1-23.

7. Uppsala Monitoring Centre. VigiBase. In: VigiBase. 2020. https ://www.who-umc.org/vigibase/vigibase/. Accessed 6 Jan 2020.
8. Uppsala Monitoring Centre. WHO Programme for International Drug Monitoring. 2020. https://www.who-umc.org/global-pharm acovigilance/who-programme-for-international-drug-monitoring I. Accessed 16 Jan 2020.

9. Ministério da Saúde. Portaria no 696, de 7 de maio de 2001Institui o Centro Nacional de Monitorização de Medicamentos (CNMM) sediado na Unidade de Farmacovigilância da Anvisa. 2001. https://bvsms.saude.gov.br/bvs/saudelegis/gm/2001/prt06 96_07_05_2001.html. Accessed Mar 2020.

10. Uppsala Monitoring Centre. Being a member of the WHO Programme for International Drug Monitoring. 2020. https://www. who-umc.org/global-pharmacovigilance/who-programme-forinternational-drug-monitoring/being-a-member/. Accessed 12 Feb 2020.

11. International Council for Harmonisation of Technical Requirements for Pharmaceuticals for Human Use - ICH. E2B(R3) Individual Case Safety Report (ICSR) specification and related files. 2018. https://ich.org/page/e2br3-individual-case-safety-repor t-icsr-specification-and-related-files. Accessed 25 Feb 2020.

12. International Council for Harmonisation of Technical Requirements for Pharmaceuticals for Human Use. ICH Association. Annual Report. Geneva: ICH; 2016. p. 2017.

13. Agência Nacional de Vigilância Sanitária. Rede Sentinela. 2020. http://portal.anvisa.gov.br/rede-sentinela-apresentacao. Accessed 4 Mar 2020.

14. Agência Nacional de Vigilância Sanitária. RDC No 4, de 10 de fevereiro de 2009 - Dispõe sobre as normas de farmacovigilância para os detentores de registro de medicamentos de uso humano. 2009. http://portal.anvisa.gov.br/legislacao\#/visualizar/2831942. Accessed 5 Mar 2020. 